Check for updates

Cite this: RSC Adv., 2019, 9, 6245

\title{
Tranexamic acid-loaded starch hemostatic microspheres
}

\author{
Huantong Su, (D) ${ }^{c}$ Shuda Wei, ${ }^{c}$ Fangping Chen, ${ }^{\text {abc }}$ Ruihua Cui ${ }^{\mathrm{c}}$ \\ and Changsheng Liu (D) abc
}

Efficacious hemostatics have significant potential for use in rapid exsanguinating hemorrhage control by emergency medical technicians or military medics nowadays. Current hemostatics focus primarily on speeding up the formation of blood clots, but inhibiting fibrinolysis is also critical for promoting coagulation and improving survival rates. Here we report a drug-loaded cross-linked microporous starch (TACMS) fabricated by loading tranexamic acid (TA) with antifibrinolytic properties into cross-linked microporous starch (CMS). The results showed that the cross-linking modification improved the mechanical properties and the particle density. The introduction of TA had no influence on water absorption of CMS. TACMS retained good physical hemostatic capacity and excellent biocompatibility. The prothrombin time (PT), activated partial thromboplastin time (APTT) and thrombin time (TT) of TACMS with $20 \mathrm{mg} \mathrm{g}^{-1}$ of TA were shortened greatly, indicating the chemical hemostasis of TACMS. TACMS demonstrated a $70 \%$ reduction in clotting time in vitro compared to CMS, which effectively inhibited the dissolution of fibrin and increased the strength of blood clots. Importantly, TACMS presented excellent hemostatic performance in rabbit ear artery injury and rabbit liver injury and even better hemostatic ability than Arista®. In conclusion, cross-linking, enzyme hydrolysis and modification of starch greatly improved absorption speed, blood uptake capacity and mechanical strength, and the introduction of TA simultaneously amplified the physical hemostasis and inhibited the dissolution of fibrin. The potent hemostatic ability of TACMS resulted from the synergistic role of physical hemostasis and drug hemostasis. The results of the present study put forward TACMS as a safe and effective hemostatic system and present a platform for

Received 8th August 2018

Accepted 17th January 2019

DOI: $10.1039 / \mathrm{c} 8 \mathrm{ra06662k}$

rsc.li/rsc-advances further optimization studies of materials with enhanced hemostatic capabilities for specific injury types.

\section{Introduction}

Uncontrolled hemorrhage, which typically involves the torso and limb junctional zones, remains a great challenge. ${ }^{1}$ The effective control of blood loss is of great significance for improved survival and a reduction in delayed causes of death from the resulting coagulopathy, infection and multisystem organ failure. ${ }^{2}$

As a complement to traditional surgical techniques, biomaterials have been developed to control life-threatening extremity hemorrhaging. ${ }^{3}$ Currently, multifarious highly efficient hemostasis biomaterials have been designed and developed. Although some have proved valuable for hemorrhage control, and some (such as HemCon ${ }^{\circledR}$, QuikClot ${ }^{\circledR}$ and Arista ${ }^{\circledR}$ ) are even available on the market, most lack efficacy against severe bleeding., ${ }^{4,5}$ Due to their huge surface area, high water absorption and good

${ }^{a}$ The State Key Laboratory of Bioreactor Engineering, East China University of Science and Technology, Shanghai 200237, P. R. China. E-mail: fpchen@ecust.edu.cn; Fax: +86-21-64251358; Tel: +86-21-64251308

${ }^{b}$ Key Laboratory for Ultrafine Materials of Ministry of Education, School of Materials Science and Engineering, East China University of Science and Technology, Shanghai 200237, P. R. China

${ }^{c}$ Engineering Research Center for Biomedical Materials of Ministry of Education, East China University of Science and Technology, Shanghai 200237, P. R. China biocompatibility, microporous polysaccharide hemispheres (MPH, Arista $\left.{ }^{\circledR}\right)$ have captured great attention and proved to be valid in several indications. However, Arista ${ }^{\circledR}$ only had an effect on control of body surface cavities and other small areas of bleeding, and it did not work on internal hemorrhages..$^{6-9}$ Obviously, Arista ${ }^{\circledR}$ achieves hemostasis based only on its physical properties, so the hemostatic effect is limited. Therefore, the ideal hemostatic should be effective, safe, convenient and inexpensive. ${ }^{\mathbf{1 0}}$

Starch has an extensive range of biomedical applications due to its excellent degradability, biocompatibility, and low cost, and it is an abundant and renewable resource. ${ }^{11,12}$ Microporous starch (MS) is a typical microporous material with high porosity, huge surface area and excellent hydrophilicity. ${ }^{13}$ More importantly, MS can rapidly absorb water, which increases the concentration of endogenous coagulation factors and platelet factors at the site of the bleeding, thus forming blood clots and possessing superior hemostatic ability. ${ }^{6}$ Combined with its inherent biosafety, simplicity of preparation and long shelf life during storage, MS has become one of the most attractive hemostatic agents.

Although it is effective in hemorrhage control for low bleeding flows, ${ }^{\mathbf{1 4}} \mathrm{MS}$ showed insufficient hemostatic capacity to stop larger and more severe bleeding. ${ }^{15,16}$ When applied to 
a massive bleeding wound, MS powder is always easily washed out by the bloodstream and exhibits lower flow ability by selfagglomeration. ${ }^{17}$ If the dispersity and the compressibility of MS are increased, the hemostatic capacity will be enhanced. Therefore, we plan to modify the MS before enzymolysis to improve its compressibility. Crosslinking improves the regularity of starch granules and the density of starch granule accumulation, so as to enhance the physical plugging property of the material to the blood. Therefore, we modified natural starch by crosslinking and then produced crosslinking microporous starch (CMS). However, CMS has an inherently limited capacity for achieving hemostasis merely by rapid absorption and swelling. From this viewpoint, the hemostatic capacity of CMS would be enhanced if it could promote the formation of fibrin or inhibit fibrin dissolution.

Blood coagulation is a dynamic process involving fibrin formation and fibrin dissolution. Fibrin is easily dissolved by fibrinolytic enzyme at the early stage of blood clotting. ${ }^{18}$ Current hemostatic materials focus primarily on speeding up the formation of blood clots but ignore the influence of the fibrinolytic system on coagulation. Inhibiting fibrinolysis is also critical for promoting coagulation. ${ }^{19}$ However, hemostatic materials combining the rapid formation of clots with effective inhibition of fibrinolysis have rarely been reported.

Tranexamic acid (TA) is a synthetic anti-fibrinolytic drug. ${ }^{20}$ Similar to lysine in structure, TA can competitively inhibit the adsorption of plasminogen and lysine binding sites on fibrin, protecting fibrin from being degraded or dissolved by fibrinolytic enzymes. ${ }^{\mathbf{1 0 , 2 0 - 2 2}}$ It is reported that TA has improved survival rates for traumatic hemorrhage. ${ }^{23} \mathrm{TA}$, a cheap, non-patented drug, has been included in the list of essential drugs by the WHO and is widely used all over the world. ${ }^{24-26}$

In this study, we first developed a TA-loaded crosslinked microporous starch (TACMS) hemostatic agent. The composite presented here aims to combine the fluid uptake and rapid clot formation of crosslinked microporous starch (CMS) with the effective fibrinolysis inhibition action of TA to create a highly advantageous hemostatic agent prototype. The primary goal of this study was to ensure that the advantageous physical hemostatic mechanism and drug hemostatic mechanism were successfully combined in the hemostatic agents. Furthermore, we evaluated the hemostatic efficiency of TACMS both in vitro and in vivo. Additionally, the biocompatibility of TACMS was investigated by hemolysis assay and cytotoxicity assay.

\section{Experiment}

\subsection{Preparation of CS, CMS and TACMS}

Native corn starch was purchased from YuanJu Biotech Co., Shanghai, China. Glucoamylase $\left(10000 \mu \mathrm{g}^{-1}\right)$ and $\alpha$-amylase $\left(2000 \mu \mathrm{g}^{-1}\right)$ were purchased from Nuohui Pharmaceutical and Chemical Co., Wuhan, China. Epichlorohydrin was purchased as a crosslinking agent from Macklin, China. Tranexamic acid (TA) was purchased from Bide Pharmatech Ltd., Shanghai, China. Deionized water was used throughout this work. All other chemicals were of analytical grade unless otherwise noted.
(1) Synthesis of CS by crosslinking. Crosslinking starch (CS) was synthesized according to a previously reported procedure ${ }^{27}$ Briefly, corn starch (200 g) was dried and added to NaCl$\mathrm{NaOH}$ solution (the concentration of $\mathrm{NaCl}$ was $50 \mathrm{~g} \mathrm{~L}^{-1}$ and the $\mathrm{pH}$ was adjusted to 13 with $2 \mathrm{M} \mathrm{NaOH})(500 \mathrm{~mL})$ in a three-neck flask $(1000 \mathrm{~mL})$. The epichlorohydrin ${ }^{28,29}(6 \mathrm{~mL})$ was dropwise added to the above mixture and stirred at $50{ }^{\circ} \mathrm{C}$ on a magnetic stirrer. After reacting for $6 \mathrm{~h}$, the $\mathrm{pH}$ was adjusted to 7.0 by $0.1 \mathrm{M}$ $\mathrm{HCl}$. CS was formed after washing with deionized water three times, filtration and vacuum freeze-drying for $24 \mathrm{~h}$.

(2) Formation of CMS by enzymolysis. Crosslinked microporous starch (CMS) was formed by CS enzymolysis with glucoamylase and $\alpha$-amylase. CS (100 g) was dried and dispersed into $\mathrm{K}_{2} \mathrm{HPO}_{4}-\mathrm{KH}_{2} \mathrm{PO}_{4}$ buffer $(400 \mathrm{~mL}, \mathrm{pH}=5.8)$ in a $600 \mathrm{~mL}$ beaker. ${ }^{30}$ The glucoamylase and $\alpha$-amylase (both $0.05 \% \mathrm{v} / \mathrm{v}$ ) at a volume ratio of $1: 4$ were mixed with the above mixture and stirred at $50{ }^{\circ} \mathrm{C}$ on a magnetic stirrer. After reaction for $6 \mathrm{~h}$, $0.1 \mathrm{M} \mathrm{NaOH}$ was added to stop hydrolysis. The starch slurry was washed with deionized water three times, centrifuged at $6000 \mathrm{rpm}$ for $10 \mathrm{~min}$ and lyophilized for $24 \mathrm{~h}$ to form CMS.

(3) Preparation of TACMS by adsorption TA. A series of concentrations of TA solution $\left(2.5,5,10,25\right.$, and $\left.50 \mathrm{mg} \mathrm{mL}^{-1}\right)$ were prepared. $10 \mathrm{~g}$ of CMS was added to the TA solutions $(20 \mathrm{~mL})$ respectively. To ensure the TA solution was fully adsorbed, CMS and TA were mixed uniformly by a vortex mixer for $24 \mathrm{~h}$, followed by lyophilization for $24 \mathrm{~h}$ to form TA-loaded CMS (TACMS). (Drug loadings are 5, 10, 20, 50, and $100 \mathrm{mg} \mathrm{g}^{-1}$, respectively.)

\subsection{Characterization of CS and CMS}

The surface morphology of the CS and CMS was observed by scanning electron microscopy (SEM, S3400, Japan) at an accelerating voltage of $15 \mathrm{kV}$. The samples were sputtered with gold for $120 \mathrm{~s}$. The phase compositions were identified by X-ray diffraction (XRD, Rigaku, Japan) using $\mathrm{Cu}-\mathrm{K} \alpha$ radiation. Data were collected from $5^{\circ}$ to $75^{\circ}$ of the scanning angle $(2 \theta)$ with a step size of $0.02^{\circ}$. The Fourier-transform infrared (FTIR) spectra were obtained using a spectrometer (Nicolet 380, Thermo, USA) from $\mathrm{KBr}$ pellets at wavelengths ranging from 4000 to $400 \mathrm{~cm}^{-1}$ at a resolution of $1 \mathrm{~cm}^{-1}$ with an average of 64 scans.

\subsection{Water absorption ratio of CS, CMS and TACMS}

The absorption ratio of the samples depended on the maximum amount of liquid per unit of material absorbed in the simulated body fluid (SBF). The samples were vacuum-dried at $60{ }^{\circ} \mathrm{C}$ overnight and the initial weights were recorded as $W_{0}$. After placing the samples on filter paper in a glass funnel, SBF was added dropwise until the solution was saturated. The weight of the sample was taken as its maximal water absorption and recorded as $W_{1}$ when the first drop of fluid fell. All experiments were repeated at least three times. The water absorption ratio was calculated according to eqn (1).

$$
\text { Water absorption ratio }=\frac{W_{1}-W_{0}}{W_{0}} \times 100 \%
$$




\subsection{Determination of the best drug loading}

The drug loading was optimized according to the clotting time (CT) of different drug loading samples in vitro and the water absorption ratio described above. Clotting time (CT) is a key parameter for identifying hemostatic performance. ${ }^{31}$ Fresh blood containing $3.8 \%$ sodium citrate was collected from New Zealand rabbits into a siliconized tube. $270 \mu \mathrm{L}$ of blood and 30 $\mu \mathrm{L}$ of $0.2 \mathrm{M} \mathrm{CaCl}_{2}$ solution were added to a $2 \mathrm{~mL}$ plastic tube and stirred for $5 \mathrm{~s}$ with a vortex shaker. $150 \mu \mathrm{L}$ of recoagulated blood was then taken out to test, and the CT was then recorded. The rest of the recoagulated blood was treated as a blank control. CT was calculated according to eqn (2). We chose the sample with the shortest CT as the best drug loading agent.

$$
\text { Percent of control }=\frac{t_{\text {sample }}}{t_{\text {control }}} \times 100 \%
$$

\subsection{APTT, PT, TT assays}

The activated partial thromboplastin time (APTT), prothrombin time (PT) and thrombin time (TT) were tested with an MC-2000 semi-automatic coagulation analyzer (TECO, Germany). Platelet poor plasma (PPP) was obtained by centrifuging the fresh blood at $3000 \mathrm{rpm}$ for $15 \mathrm{~min}$ at $4{ }^{\circ} \mathrm{C}$. All the test samples, test reagents and $\mathrm{CaCl}_{2}$ solution were incubated at $37^{\circ} \mathrm{C}$ in advance.

To test APTT, $100 \mu \mathrm{L}$ of PPP and $100 \mu \mathrm{L}$ of APTT reagent were incubated at $37{ }^{\circ} \mathrm{C}$ for $5 \mathrm{~min}$, and then $100 \mu \mathrm{L}$ of $\mathrm{CaCl}_{2}$ was added to the tube. The PT test was performed by adding $50 \mu \mathrm{L}$ of PPP and $100 \mu \mathrm{L}$ of PT reagents to the tube successively. The test for TT is similar to that for PT; $100 \mu \mathrm{L}$ of TT reagents and $100 \mu \mathrm{L}$ of PPP were added to the tube containing the materials. Pure APTT, PT and TT reagents without samples were taken as negative controls, and Arista ${ }^{\circledR}$ was a positive control. All the experiments were run in triplicate $(n=3)$.

\subsection{TEG assay}

The clotting activities of blood exposed to hemostatic powders were analyzed by TEG (thromboelastogram, TEG-5000®, Medical Technology Co., Ltd. Shanxi Yu Zeyi) assays in vitro. TEG characterizes the formation and strength of a blood clot as a function of time. ${ }^{32}$ The test procedure for TEG is as follows: First, $20 \mathrm{mg}$ of each sample were placed in small plastic vials; $1 \mathrm{~mL}$ of blood was then added and mixed uniformly; finally, a $340 \mu \mathrm{L}$ mixture of blood and sample was placed in TEG cups for analysis. $20 \mu \mathrm{L}$ of $\mathrm{CaCl}_{2}(0.2 \mathrm{M})$ were added to the cups before adding the blood samples to avoid anticoagulation. The test was measured at $37^{\circ} \mathrm{C}$. A recalcified blood sample without further treatment was chosen as a negative control. All of the following variables were recorded: reaction time ( $r$, minutes), clotting time ( $K$, minutes), angle ( $\alpha$, degrees), and maximum amplitude (MA, millimeters).

\subsection{Fibrin formation and red blood cell adhesion}

The fibrin formation of PPP on the sample surface was observed by a scanning electron microscope (SEM). PPP $(50 \mu \mathrm{L})$ along with CMS or TACMS (50 mg) and $5 \mu \mathrm{L}$ of $\mathrm{CaCl}_{2}(0.2 \mathrm{M})$ were added to the 24 -well culture plate and incubated at $37^{\circ} \mathrm{C}$ for $30 \mathrm{~min}$. To remove the non-coagulated plasma, the samples were then dip rinsed twice in phosphate buffered solution (PBS). The samples were fixed with $2.5 \%$ glutaraldehyde solution $(1 \mathrm{~mL})$ for $15 \mathrm{~min}$ and dehydrated by a graded series of ethanol solutions. After drying for $12 \mathrm{~h}$ at $37{ }^{\circ} \mathrm{C}$, the samples were observed by SEM. The red cells were obtained by centrifuging the whole blood at $1500 \mathrm{rpm}$ for $5 \mathrm{~min}$ at $4{ }^{\circ} \mathrm{C}$, then $\operatorname{dip}$ rinsing twice in PBS. The red cell solution was prepared by dispersing the red cells in PBS $(\mathrm{v} / \mathrm{v}=1: 1)$. The red blood cell adhesion was observed as in the above method.

\subsection{Hemolysis assay}

Hemolysis assays were used to evaluate the interaction between blood and materials. ${ }^{33}$ For the test, the red blood cells were obtained as a red blood cell adhesion. The red blood cells were dispersed in PBS at a ratio of $1: 7$. Each sample was $5 \mathrm{mg}$ in weight and incubated with $1 \mathrm{~mL}$ of red cell dispersion for $60 \mathrm{~min}$ at $37^{\circ} \mathrm{C}$. All the test tubes containing test matters were centrifuged at $3000 \mathrm{rpm}$ for $10 \mathrm{~min}$, from which supernatant was extracted. An ultraviolet spectrophotometer was used to determine the OD value of the supernatant at $540 \mathrm{~nm}$. The hemolysis ratio was calculated with eqn (3). Three parallel items were set for each group.

$$
\text { Hemolysis ratio }=\frac{\mathrm{OD}_{540, \text { sample }}-\mathrm{OD}_{540, \mathrm{PBS}}}{\mathrm{OD}_{540, \mathrm{H}_{2} \mathrm{O}}-\mathrm{OD}_{540, \mathrm{PBS}}} \times 100 \%
$$

\subsection{Cytotoxicity assay}

The cytotoxicity of the prepared samples (CMS, TACMS) was investigated by MTT assay. A mouse myoblast cell line (ACTT, C2C12) was used. In short, C2C12 cells were cultured in Dulbecco's Modified Eagle's Medium (DMEM) with $0.11 \mathrm{~g} \mathrm{~L}^{-1}$ L-glutamine, $2.2 \mathrm{~g} \quad \mathrm{~L}^{-1}$ sodium bicarbonate, $10 \%$ fetal bovine serum and $2 \%$ antibiotics $\left(200 \mathrm{mg} \mathrm{mL}^{-1}\right.$ penicillin and $200 \mathrm{mg} \mathrm{mL} \mathrm{mL}^{-1}$ streptomycin) for 7 days. The medium was changed once every three days. The cells were seeded into 96well plates at a density of 5000 cells per well and were then exposed to leaching solutions of the tested samples at a concentration of $0.1-0.5 \mathrm{mg} \mathrm{mL}^{-1}$. After incubation in a fully humidified atmosphere of $5 \% \mathrm{CO}_{2}$ at $37{ }^{\circ} \mathrm{C}$ for 1 day, the cell viabilities were assessed by MTT assays. The results were reported as the means of at least five wells and presented as the cell viability of cells relative to the negative control.

\subsection{Hemostasis evaluation in vitro and in vivo}

All animal experiments were approved by the ethical committee of the National Tissue Engineering R\&D Center (Shanghai, China). All animals were cared for and treated in accordance with the National Research Council's Guide for the Care and Use of Laboratory Animals. All efforts were made to minimize suffering and the number of animals used.

Surgery was completed on $2.5-3 \mathrm{~kg}$ male New Zealand white rabbits (Jambo Biological Technology Co. Ltd, Shanghai, China). Rabbits were only supplied with water $12 \mathrm{~h}$ before the operation 
and received general anesthesia with pentobarbital sodium $(30 \mathrm{mg}$ $\mathrm{kg}^{-1}$ ) by intravenous injection before the surgery. Samples were sterilized by ultraviolet radiation (Heming Co, Ltd, Shanghai, China). All procedures were conducted under sterile conditions. 20 rabbits were divided into four groups (CS, CMS, TACMS and Arista ${ }^{\circledR}$ group) for hemostasis injury. Each rabbit was operated on to produce a liver injury model and an ear artery injury.

(1) Hemostasis in rabbit ear artery model. For ear artery hemostasis surgery, a slotted wound of $1 \mathrm{~cm}$ with a depth of $0.3 \mathrm{~cm}$ was made in the ear artery using a scalpel, and the transudatory blood on the wound surface was removed with gauze. CS, CMS, TACMS or Arista ${ }^{\circledR}$ were sprayed directly onto the wound, and pressure applied gently with gauze for $10 \mathrm{~s}$; the time of bleeding was recorded (Fig. 7). After accomplishment of hemostasis, the samples were scrubbed and the wound was washed with physiological sodium chloride solution. To determine the total blood loss of the rabbits, the weights of sterile gauze before and after surgery were measured. The rabbits were monitored for 3 days, 7 days and 42 days to evaluate their survival rate. All experiments were carried out five times $(n=5)$.

(2) Hemostasis in rabbit liver model. For live hemostasis surgery, the process is similar to the ear artery model except for the wound type. A cross-shaped wound of $2 \times 2 \mathrm{~cm}$ with a depth of $0.5 \mathrm{~cm}$ was made in the liver using a scalpel. Drugs were sprinkled onto the wound before the wound was sutured layer after layer with monofilament nylon.

\subsection{Statistical analysis}

Results were expressed as means \pm standard deviations. Except for special requests in the animal experiments, all the data were generated using more than three independent experiments. Statistical analysis was conducted using Origin 8.0.

\section{Results and discussion}

\subsection{CS and CMS characterization}

Due to its excellent porosity structure, high adsorption and biocompatibility, MS has been widely applied as a biomedical material. Crosslinked microporous starch (CMS) as a typical microporous material was successfully manufactured via the technique mentioned above.
FTIR spectroscopy analysis was conducted to determine the chemical changes before and after the chemical modification. Due to the chemical bonds of NS, CS and CMS being largely the same, there is no difference in the absorption wavenumber. However, the shape and the intensity of the absorption peaks at $3394 \mathrm{~cm}^{-1}, 1631 \mathrm{~cm}^{-1}$, and $1600 \mathrm{~cm}^{-1}$ are different, as shown in Fig. 1(a). Compared with NS, those of CS and CMS have increased width at $3390 \mathrm{~cm}^{-1}$ with increasing asymmetry, indicating that they are associated with the $-\mathrm{OH}$ bond of the starch granules. The intensity of the absorption peaks at $1631 \mathrm{~cm}^{-1}$ and $1600 \mathrm{~cm}^{-1}$ of CS and CMS is decreasing, which is due to the crosslinking of starch particles when the hydrogen bonds are replaced by crosslinking bonds.

The XRD patterns of NS, CS and CMS are displayed in Fig. 1(b). NS has the characteristic A-type pattern with major peaks at $15^{\circ}, 17^{\circ}, 18^{\circ}$ and $23^{\circ},{ }^{13}$ while the peaks of CS and CMS were weak at the same positions. This indicated that the crystalline structures of NS were mostly damaged after the crosslinking reaction, and the crystallinity of CS and CMS became lower.

As previously described, we prepared CS through a crosslinking reaction of NS, and then through an enzymolysis reaction to prepare CMS. As shown in Fig. 2, NS is spherical with a smooth surface, with some edges and corners, but without holes. After crosslinking, most starch granules remained spherical, but the surface of CMS became rough and porous. These were not only beneficial to the interactions between the sample and the body fluid but were good for the tranexamic acid loading as well.

\subsection{Determination of the optimal drug loading}

The adsorption capacity of hemostatic agents contributes to a concentration of the blood tangible composition, such as platelets, red blood cells and coagulation factors. ${ }^{34}$ Therefore, an excellent adsorption capacity is crucial to hemostasis. So, we tested the water absorption and clotting time of the samples loaded with different dosages of TA to determine the drug loading of the material that has the best hemostatic capacity.

In order to investigate the absorption quantitatively, the swelling properties of the samples were tested by immersion in $\mathrm{SBF}(\mathrm{pH}=7.4)$ for $30 \mathrm{~min}$ and the results are presented in
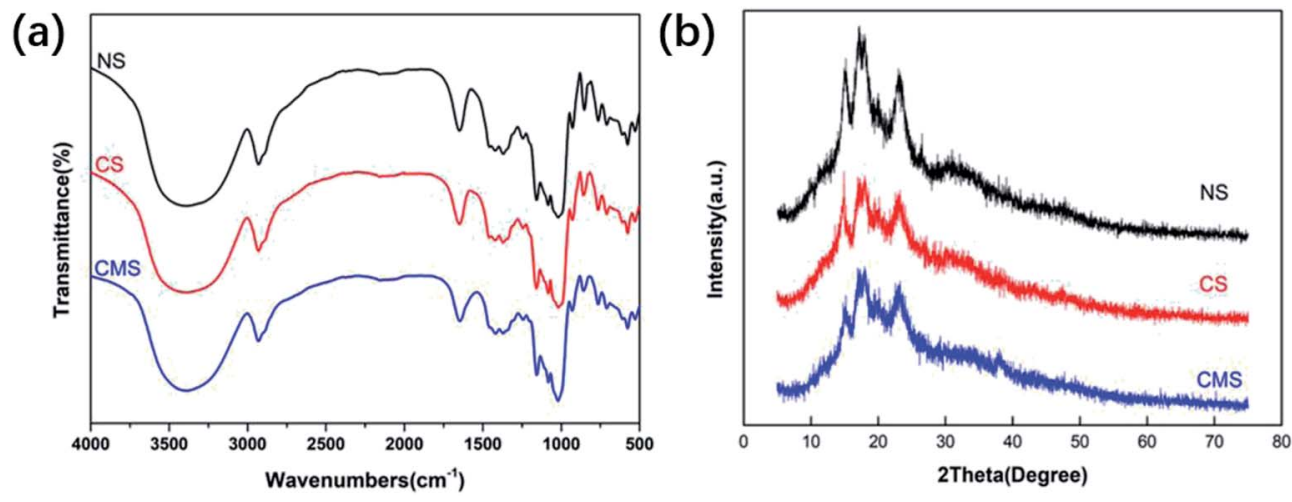

Fig. 1 (a) FTIR spectra of NS, CS and CMS. (b) XRD patterns of NS, CS and CMS. 

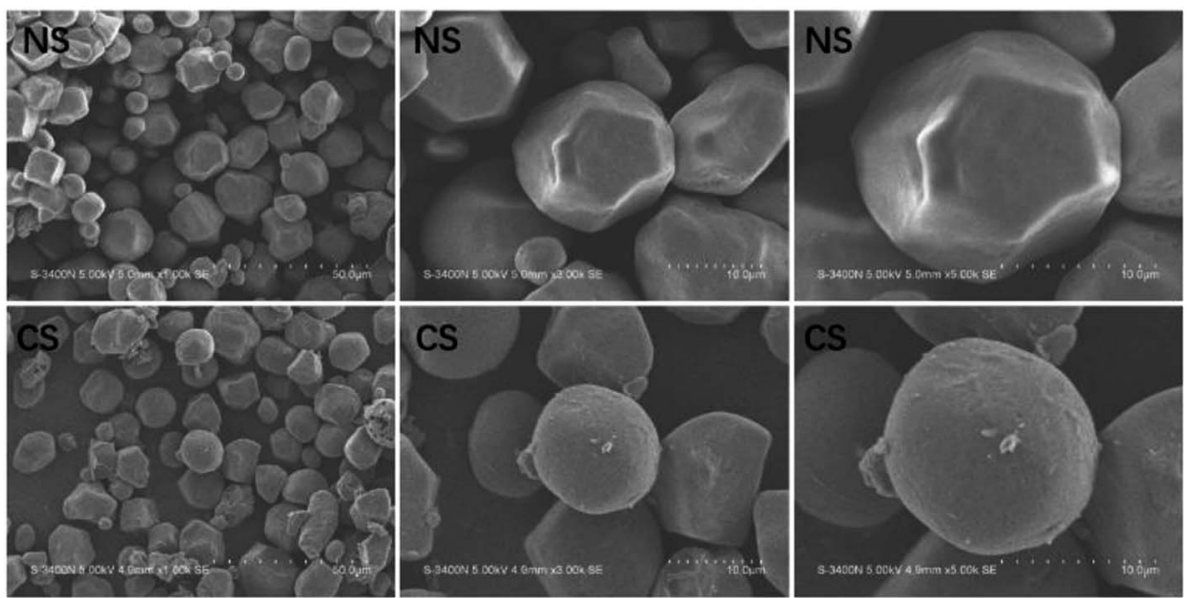

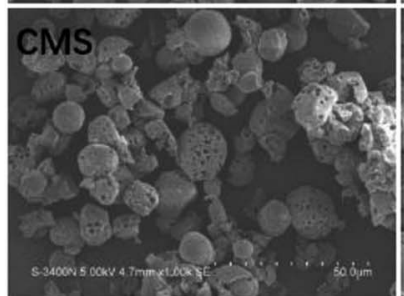

$1000 \mathrm{X}$

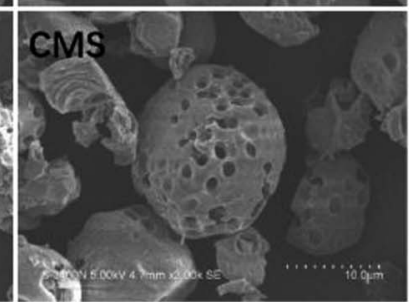

$3000 X$

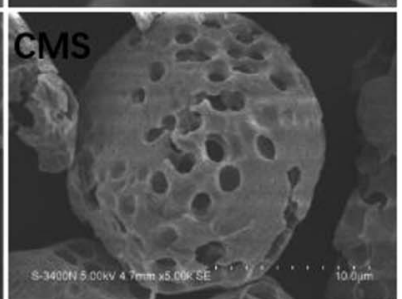

$5000 X$

Fig. 2 SEM micrographs of NS, CS and CMS.

Fig. 3(a). In detail, the absorption ratios of CS (115.1\%) and CMS $(169.5 \%)$ are much higher than that of NS $(81.92 \%)$. The introduction of tranexamic acid had no influence on the absorption ratio of the samples when the drug load was less than $20 \mathrm{mg} \mathrm{g}^{-1}$. The absorption ratio became lower when the drug load went up to $50 \mathrm{mg} \mathrm{g}^{-1}$.

CT was applied to evaluate the hemostatic potential of the samples. As shown in Fig. 3(b), it could be seen that the addition of the materials greatly shortened $\mathrm{CT}$ at different levels. When TA is added to the CMS, the clotting time is similar to that of CMS, indicating that the addition of TA preserves the physical hemostatic effect of CMS. We speculate that TA occupies the voids on the CS surface and reduces the specific surface area of the particles. Water absorption decreases and hinders the physical hemostasis of the materials. Therefore, CMS had a shorter clotting time than most of the TACAMSs samples. According to the results, TACMS-20 had the shortest clotting time, in agreement with the absorption ratio results. Therefore, we chose $20 \mathrm{mg} \mathrm{g}^{-1}$ as the optimal drug loading in the following study.

\subsection{Hemostatic effect in vitro}

The clot strength as well as other clotting kinetics parameters are listed in Table 1. It was obvious that the $r$ values of CMS and TACMS were shorter than that of the control group. CMS can absorb water quickly, concentrating the complex of coagulation factor VIIa and tissue factor, and activating coagulation factor XII to produce protein hydrolase FXIIa. At the same time, CMS adsorbed the calcium in the blood electrostatically, quickly triggering the internal and external coagulation pathway. The $r$
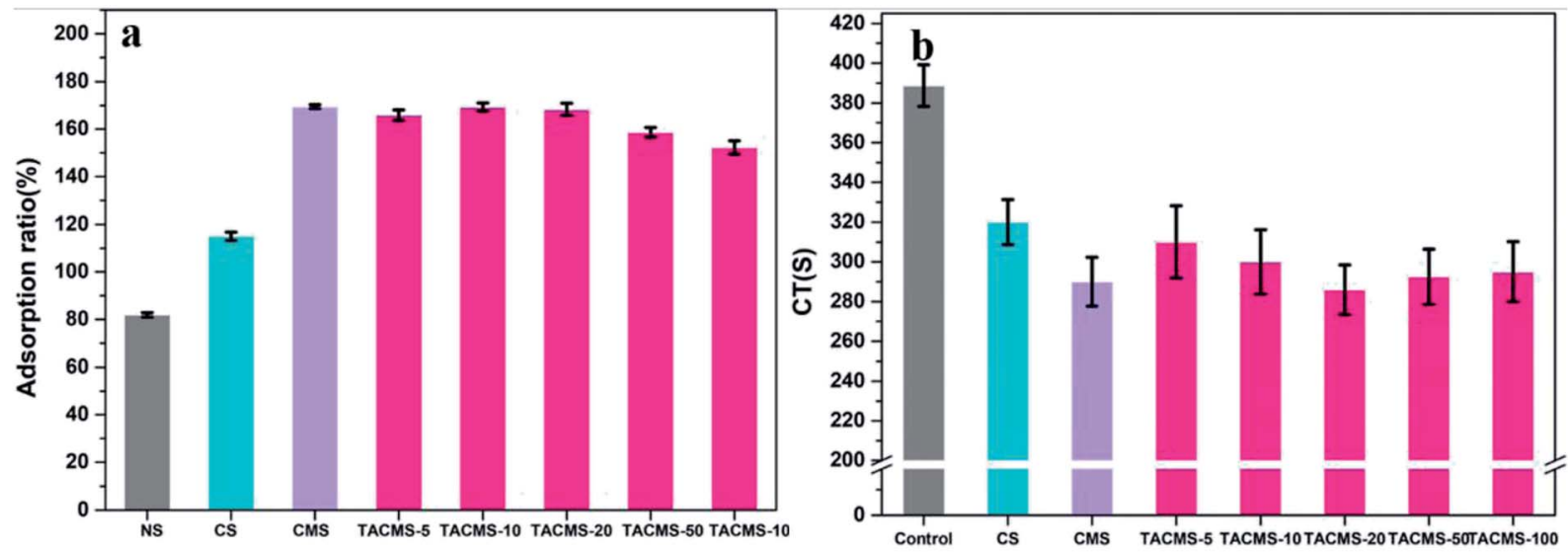

Fig. 3 (a) The absorption ratios of NS, CS, CMS and TACMS. (b) CT of CMS and TACMS. 
Table 1 Clotting kinetics parameters of blood mixed with CMS and TACMS

\begin{tabular}{lclll}
\hline Material & $r(\min )$ & $K(\min )$ & $\alpha(\mathrm{deg})$ & $\mathrm{MA}(\mathrm{mm})$ \\
\hline Reference range & $5-10$ & $1-3$ & $53-72$ & $50-70$ \\
Control & 12.1 & 3.5 & 48.7 & 62.6 \\
CMS & 5.1 & 2.1 & 62.3 & 64.2 \\
TACMS & 4.9 & 1.7 & 67.1 & 66.2 \\
\end{tabular}

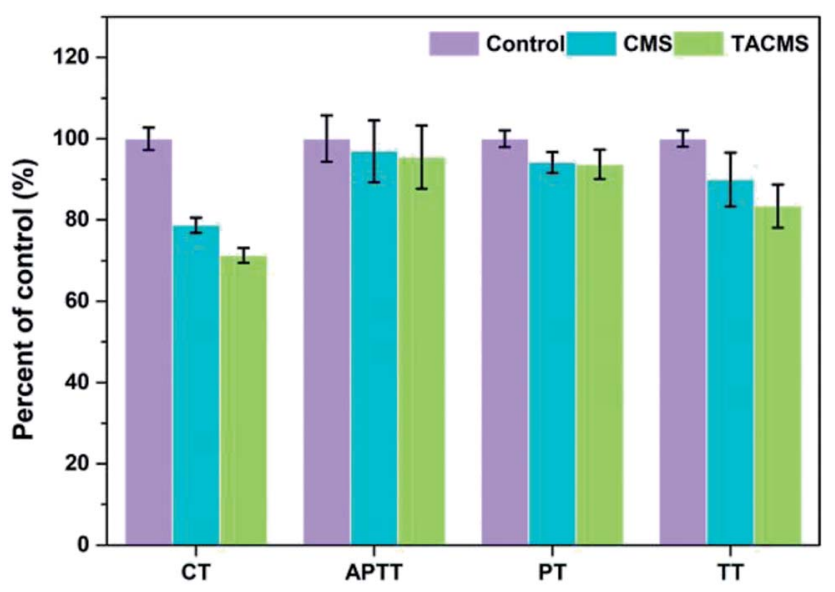

Fig. 4 Hemostatic effect in vitro (CT, APTT, PT and TT).

value is the reaction time, from the beginning of clotting to the formation of fibrin. This showed that CMS and TACMS have the effect of shortening the initial clotting time. Compared with the control group, the MA values of CMS and TACMS increased gradually. The MA value is the maximum blood clot strength during the whole coagulation process. This can be attributed to the adhesion of red cells to CMS and TACMS (Fig. 5(c and d)), which was beneficial to the formation of red clots and increased the strength of the blood clot. All of these proves that the introduction of tranexamic acid gave TACMS the effect of antifibrinolysis (Fig. 5(a and b)), which increased the strength of the blood clot, increasing the MA value.

CT was used to evaluate the hemostatic potential of the samples. The blood coagulation cascade consists of the intrinsic pathway, the extrinsic pathway and the common pathway of coagulation. APTT, PT and TT were used to characterize the influence of CMS and TACMS on the intrinsic pathway, extrinsic pathway and common pathway, respectively. Those coagulation parameters are all shown in Fig. 4. TACMS changed these four parameters most obviously. For APTT and PT, TACMS showed no significant difference to CMS. However, the CT and TT of TACMS dropped to $71.34 \%$ and $83.41 \%$, respectively, taking the control as $100 \%$. The samples loaded with TA effectively inhibited fibrin dissolution and accelerated the formation of a three-dimensional protein network (Fig. 5(a and b)), thereby promoting coagulation and shortening CT and TT.

With the help of micropores on the surface, CMS and TACMS acted as molecular sieves, rapidly adsorbing a large amount of water, concentrating blood tangible composition and accelerating blood coagulation. In fact, there were hardly any cells adhering to CMS (Fig. 5(c)). However, more red blood cells were found on the surface of TACMS (Fig. 5). This may be related to the absorption of TA onto TACMS. The protein network promoted the adhesion of red blood cells on the surface of TACMS. Fig. 5(a and b) further confirm fibrin formation on the surface of CMS and TACMS. Furthermore, many more fibrins were observed on the surface of TACMS than on CMS. This indicated that the
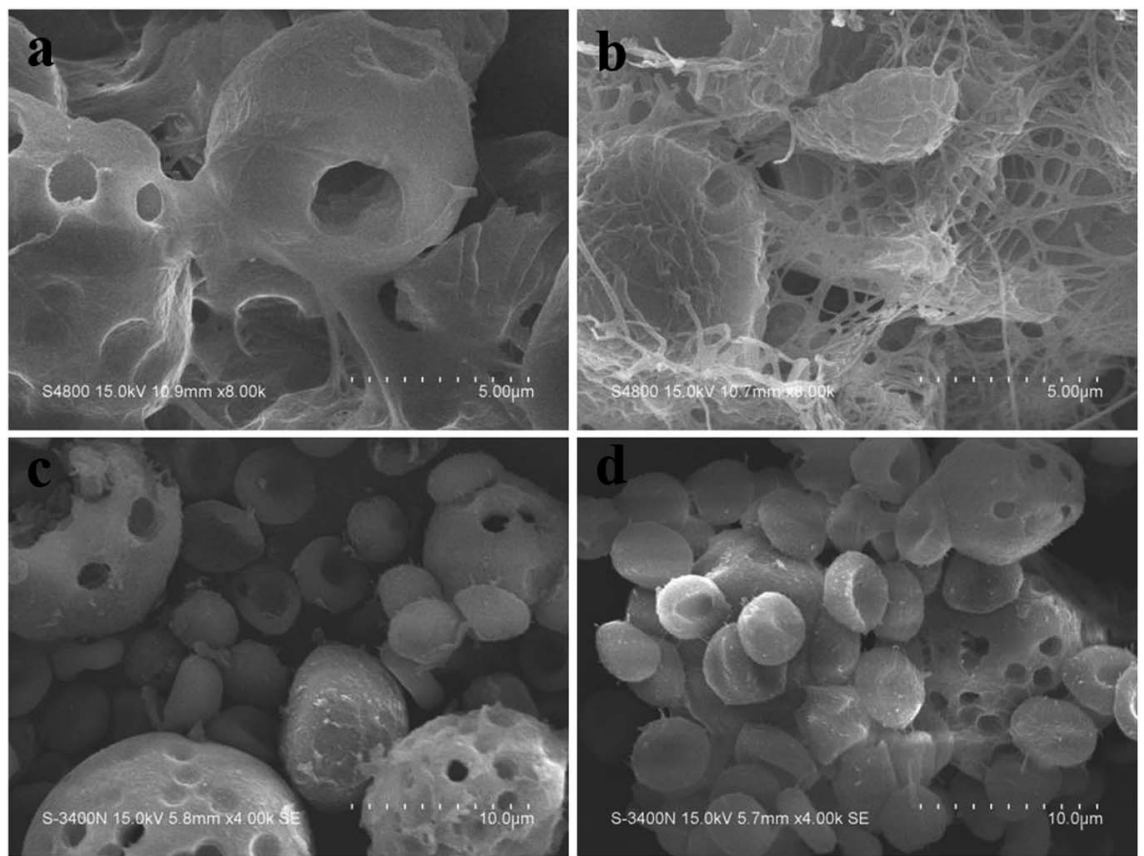

Fig. 5 Fibrin formation on the surface of materials (a) CMS, (b) TACMS and the red blood cells adhered to CMS (c) and TACMS (d). Scale bars represent $5 \mu \mathrm{m}$ ( $a$ and $b$ ) and $10 \mu \mathrm{m}$ (c and d). 

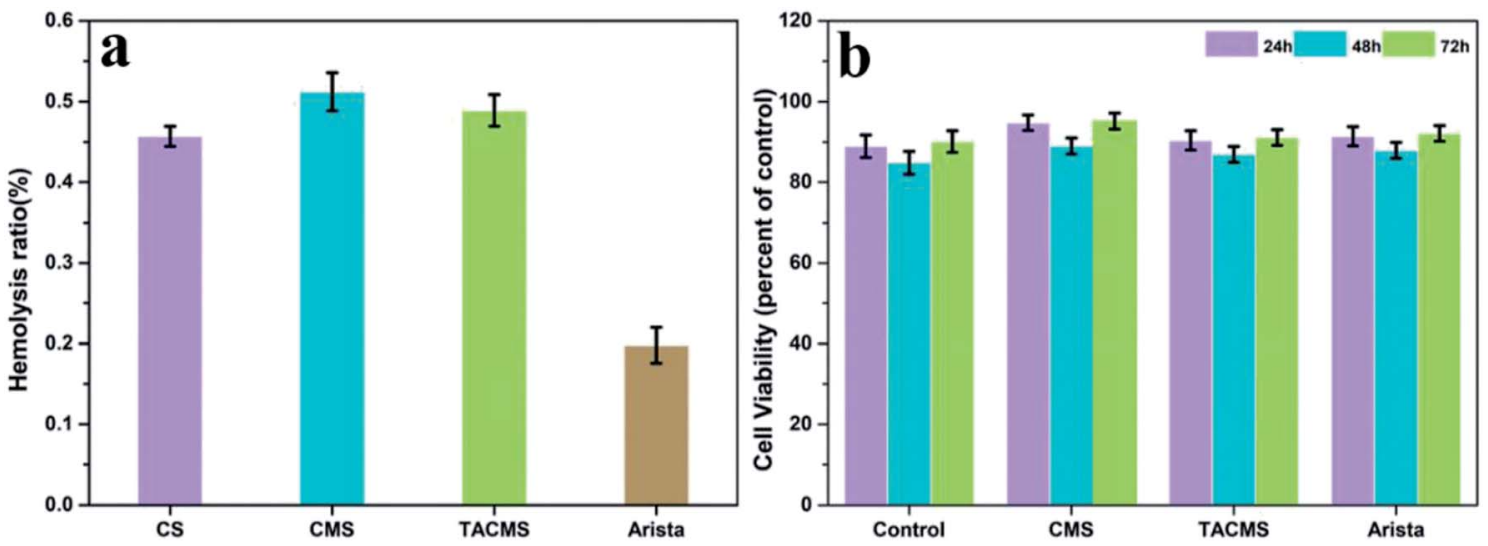

Fig. 6 (a) Hemolysis ratio and (b) MTT assay Arista ${ }^{\circledR}$ of CS, CMS, TACMS and Arista ${ }^{\circ}$.

introduction of TA effectively inhibited fibrin dissolution. All of these were consistent with the analysis from TEG above. In addition, the red blood cells adhering to the samples were in good condition, indicating their superior cytocompatibility.

\subsection{Hemolysis and cytotoxicity evaluation}

Hemolysis assay is based mainly on the degree of erythrolysis and hemoglobin dissociation when the materials are in contact with blood. It is an easy and reliable method to evaluate the blood compatibility of materials. According to Standardization Administration of the People's Republic of China, samples with a hemolysis ratio less than $5 \%$ meet the hemolysis requirement for medical application. Fig. 6(a) shows that the hemolysis ratios of CS (0.457\%), CMS (0.512\%), TACMS (0.489\%) and Arista ${ }^{8}(0.198 \%)$ were much less than $5 \%$. The results indicate that all four samples were nontoxic and had no side effects on red blood cells.

An MTT assay was conducted to further examine the biocompatibility of the samples. Fig. 6(b) shows the cell viability of CMS, TACMS and Arista ${ }^{\circledR}$ after incubating with $\mathrm{C} 2 \mathrm{C} 12$ for $24 \mathrm{~h}, 48 \mathrm{~h}$ and $72 \mathrm{~h}$. No significant difference in cytotoxicity was seen among all the groups. In particular, the cell viability of TACMS showed no significant difference to Arista ${ }^{\circledR}$. Cell viabilities remained at a high level when the culture time was extended from 24 hours to 72 hours. The results indicated that TACMS had good biocompatibility as a promising hemostasis agent.

\subsection{Hemostatic efficiency in vivo}

The ear artery injury of rabbits was surgically induced as described above, and treated with CS, CMS, TACMS or Arista ${ }^{\circledR}$ (Fig. 7). The time to complete hemostasis and bleeding volume in the different groups were recorded. After $138 \pm 8 \mathrm{~s}$ (CMS group, $n=5$ ) and $108 \pm 5 \mathrm{~s}$ (TACMS group, $n=5$ ) complete hemostasis was achieved while the Arista ${ }^{\circledR}$ group needed $120 \pm$ $3 \mathrm{~s}$. However, the control group (CS group, $n=5$ ) took a prolonged time of $190 \pm 7 \mathrm{~s}$ to complete hemostasis.

Statistically significant differences were found among all the CS, CMS and TACMS groups. Also, the bleeding volume and hemostasis time of TACMS were similar to those of the Arista ${ }^{\circledR}$ group. These results indicated that the crosslinked microporous starch (both CMS and TACMS) had an effect on complete hemostasis in ear vein injuries. Furthermore, the hemostasis time of TACMS was shorter than that for CMS. The result further indicated that TACMS displayed a superior hemostatic performance (the least time to hemostasis and the least bleeding volume) in ear vein injuries.

Fig. 8 shows the treatment process for rabbit liver injury. All the samples contacted blood completely when they were added into the wounds. Robust clots were seen to form with the samples, with platelets and clotting proteins tightly adhering to the bleeding surface. Fig. 8(e) shows the time to complete hemostasis and bleeding volume of all samples. The times to
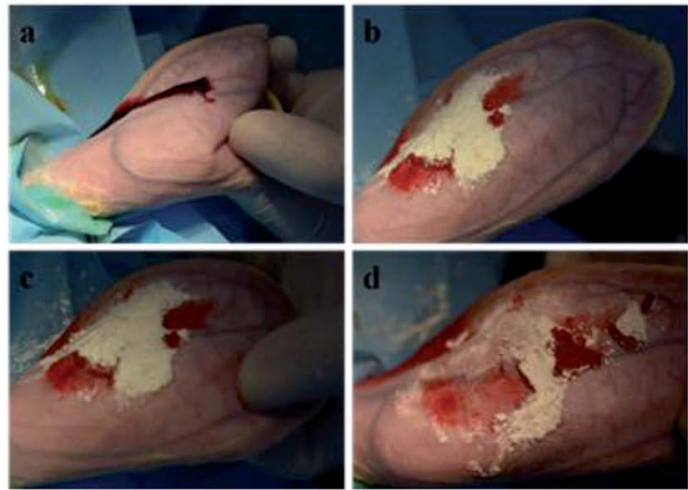

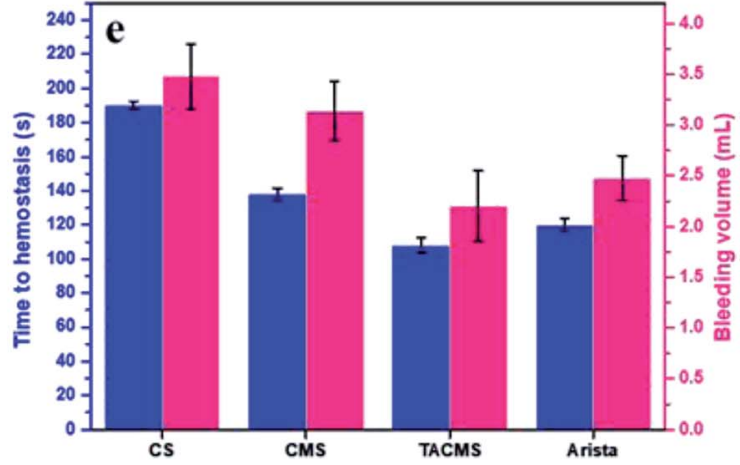

Fig. 7 Hemostatic capacity evaluation in rabbit ear artery injury: (a) bleeding after trauma. (b) Applying the hemostatic materials on the wound. (c) Hemostatic process. (d) Complete hemostasis. (e) Bleeding volume and hemostasis time in rabbit ear artery injury. 

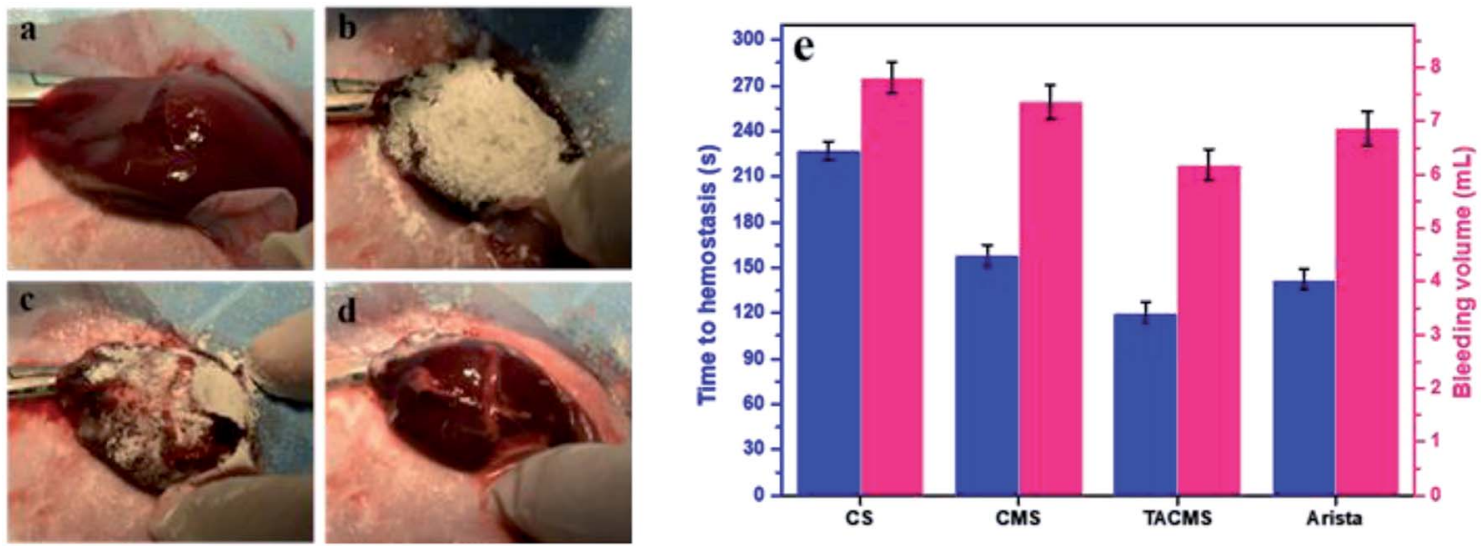

Fig. 8 Hemostatic capacity evaluation in rabbit liver injury: (a) bleeding after trauma. (b) Applying the TACMS on the wound. (c) Hemostatic process. (d) Complete hemostasis. (e) Bleeding volume and hemostasis time in rabbit liver injury.
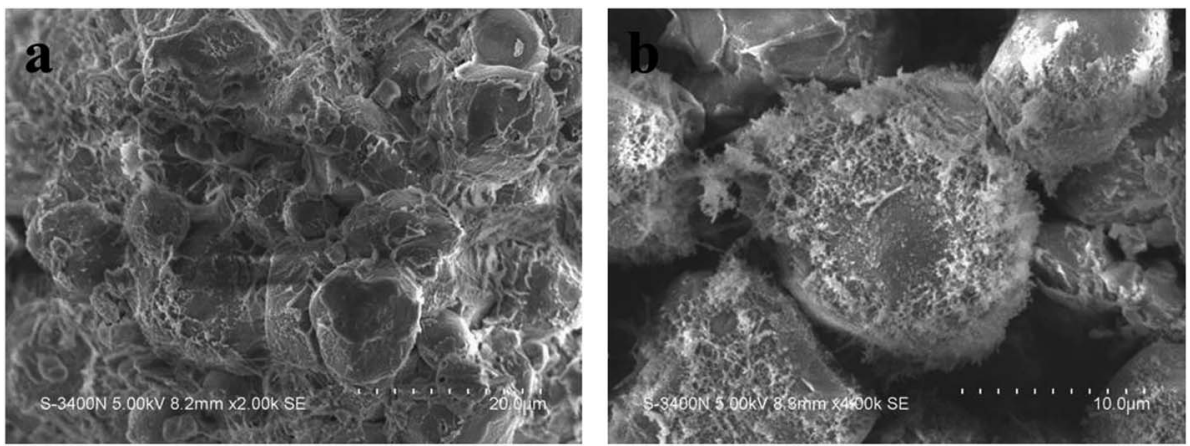

Fig. 9 SEM images of TACMS after hemostasis. (a) 2000x, (b) 4000x.

form blood clots on CS and CMS were about $227 \pm 10 \mathrm{~s}$ and 158 \pm 7 s, respectively. The blood clots of TACMS formed within 120 $\pm 6 \mathrm{~s}$, which was shorter than for the Arista ${ }^{\circledR}$ group (142 $\pm 3 \mathrm{~s}$ ). Statistically significant differences were found among all the CS, CMS and TACMS groups. These results indicated that TACMS helped to fasten complete hemostasis, displaying a superior hemostatic performance (least time to hemostasis and least bleeding volume) in liver injury.

All the samples effectively controlled the bleeding and TACMS showed the best hemostatic performance.

Fig. 9 reveals the SEM morphologies of TACMS interacting with blood. A number of blood cells were seen to closely agglutinate on TACMS. Furthermore, many fibrins were formed on TACMS and were closely bound to the blood cells around the starch microsphere like cages.

\section{Conclusion}

TACMS has been successfully developed to control hemorrhaging, and its hemostatic efficacy together with good biocompatibility were evaluated. The results showed that TACMS could not only quickly and effectively absorb fluid but also inhibited fibrinolysis and induced aggregation of RBCs. The synergistic effects of the inhibition of fibrinolysis and physical absorption endowed TACMS with potent hemostatic properties. The fabricated TACMS, with a combination of improved hemostatic efficiency and excellent biocompatibility, should be a strong candidate for controlling hemorrhaging. Research is ongoing to explore the molecular mechanism of their hemostatic activity and other chemical modifications to enhance the hemostatic efficiency of CMS.

\section{Conflicts of interest}

There are no conflicts to declare.

\section{Acknowledgements}

This investigation was supported by the National Key Research and Development Program of China under Grant No. 2016YFC1102900, the National Natural Science Foundation of China under Grant No. 31370960, and the Shanghai Pujiang Program under Grant 16PJD015 and Joint Fund of the Ministry of Education under Grant 6141A02022618.

\section{References}

1 C. N. Sambasivan, D. Cho, K. A. Zink, J. A. Differding and M. A. Schreiber, Am. J. Surg., 2009, 197, 576-580. 
2 C. L. Dai, Y. Yuan, C. S. Liu, J. Wei, H. Hong, X. S. Li and X. H. Pan, Biomaterials, 2009, 30, 5364-5375.

3 A. E. Pusateri, J. B. Holcomb, B. S. Kheirabadi, H. B. Alam, C. E. Wade and K. L. Ryan, J. Trauma: Inj., Infect., Crit. Care, 2006, 60, 674-682.

4 B. S. Kheirabadi, E. M. Acheson, R. Deguzman, J. L. Sondeen, K. L. Ryan, A. Delgado, E. J. Dick and J. B. Holcomb, J. Trauma: Inj., Infect., Crit. Care, 2005, 59, 25-34.

5 D. A. Hickman, C. L. Pawlowski, U. D. S. Sekhon, J. Marks and A. S. Gupta, Adv. Mater., 2018, 30, 1700859.

6 J. L. Antisdel, J. L. West-Denning and R. Sindwani, Otolaryngol.-Head Neck Surg., 2009, 141, 353-357.

7 M. Galarza, O. P. Porcar, R. Gazzeri and J. F. Martinez-Lage, World Neurosurg., 2011, 75, 491-494.

8 E. Lekniute, L. Peciulyte, R. Klimaviciute, J. Bendoraitiene and A. Zemaitaitis, Colloids Surf., A, 2013, 430, 95-102.

9 H. Peng, S. Zhong, Q. Lin, X. Yao, Z. Liang, M. Yang, G. Yin, Q. Liu and H. He, Carbohydr. Polym., 2016, 138, 210-214.

10 I. S. Ciric, R. W. Crowley, A. S. Dumont, C. Heilman and G. Schackert, Neurosurgery, 2008, 63, 372.

11 M. E. Gomes, J. S. Godinho, D. Tchalamov, A. M. Cunha and R. L. Reis, Mater. Sci. Eng., C, 2002, 20, 19-26.

12 A. Pourjavadi, A. A. Ebrahimi and S. Barzegar, J. Drug Delivery Sci. Technol., 2013, 23, 511-517.

13 F. P. Chen, X. Y. Cao, X. L. Chen, J. Wei and C. S. Liu, J. Mater. Chem. B, 2015, 3, 4017-4026.

14 R. Sindwani, Otolaryngol.-Head Neck Surg., 2009, 140, 262263.

15 K. Bjorses and J. Holst, Eur. J. Vasc. Endovasc. Surg., 2007, 33, 363-370.

16 H. B. Alam, G. B. Uy, D. Miller, E. Koustova, T. Hancock, R. Inocencio, D. Anderson, O. Llorente and P. Rhee, J. Trauma: Inj., Infect., Crit. Care, 2003, 54, 1077-1082.

17 H. Y. Zhou, C. W. Wang, H. Y. Niu, B. Duan, X. Y. Ma, H. Hong, Y. Yuan and C. S. Liu, J. Biomed. Nanotechnol., 2018, 14, 649-661.

18 J. C. Chapin and K. A. Hajjar, Blood Rev., 2015, 29, 17-24.
19 P. A. von dem Borne, L. M. P. Cox and B. N. Bouma, Blood Coagulation Fibrinolysis, 2006, 17, 251-257.

20 J. R. Baylis, A. E. St John, X. Wang, E. B. Lim, M. L. Statz, D. Chien, E. Simonson, S. A. Stern, R. T. Liggins, N. J. White and C. J. Kastrup, Shock, 2016, 46, 123-128.

21 L. W. Chan, N. J. White and S. H. Pun, ACS Biomater. Sci. Eng., 2016, 2, 403-408.

22 E. Yaniv, J. Shvero and T. Hadar, Am. J. Rhinol., 2006, 20, 227229.

23 V. Casati, L. Sandrelli, G. Speziali, G. Calori, M. A. Grasso and S. Spagnolo, J. Thorac. Cardiovasc. Surg., 2002, 123, 1084-1091.

24 C. Y. Xi, L. G. Zhu, Y. Zhuang, S. F. Wang, G. X. Sun, Y. Q. Liu and D. Q. Wang, Clin. Appl. Thromb./Hemostasis, 2018, 24, 279-286.

25 C. Zentai, P. E. J. van der Meijden, T. Braunschweig, N. Hueck, M. Honickel, H. M. H. Spronk, R. Rossaint and O. Grottke, Anesth. Analg., 2016, 123, 38-48.

26 T. F. Slaughter, Ann. Thorac. Surg., 1999, 68, 2256-2257.

27 Y.-y. Fang, L.-j. Wang, D. Li, B.-z. Li, B. Bhandari, X. D. Chen and Z.-h. Mao, Carbohydr. Polym., 2008, 74, 379-384.

28 Z. Souguir, E. About-Jaudet, L. Picton and D. L. Cerf, Drug Delivery Lett., 2012, 2, 240-250.

29 A. Kolman, M. Chovanec and S. Osterman-Golkar, Mutat. Res., 2002, 512, 173-194.

30 F. Chen, X. Cao, J. Yu, H. Su, S. Wei, H. Hong and C. Liu, Colloids Surf., B, 2017, 159, 937-944.

31 A. K. Gaharwar, R. K. Avery, A. Assmann, A. Paul, G. H. McKinley, A. Khademhosseini and B. D. Olsen, ACS Nano, 2014, 8, 9833-9842.

32 W. Zhang, D. G. Zhong, Q. Liu, Y. Zhang, N. Li, Q. Wang, Z. H. Liu and W. Xue, J. Biomater. Sci., Polym. Ed., 2013, 24, 1549-1563.

33 B. C. Evans, C. E. Nelson, S. S. Yu, K. R. Beavers, A. J. Kim, H. Li, H. M. Nelson, T. D. Giorgio and C. L. Duvall, J. Vis. Exp., 2013, 73, e50166.

34 D. Li, P. Li, J. Zang and J. Liu, J. Biomed. Biotechnol., 2012, 2012, 981321. 